\title{
Neoadjuvant immunotherapy with resectable non-small cell lung cancer: recent advances and future challenges
}

\author{
Sijia Ren ${ }^{1}$, Chunguo Wang ${ }^{2}$, Jianfei Shen ${ }^{2}$, Chengchu Zhu ${ }^{2}$ \\ ${ }^{1}$ Department of Cardiothoracic Surgery, Taizhou Hospital of Zhejiang Province, Zhejiang University School of Medicine, Taizhou 317000, China; \\ ${ }^{2}$ Department of Cardiothoracic Surgery, Taizhou Hospital of Zhejiang Province, Taizhou 317000, China \\ Correspondence to: Jianfei Shen; Chengchu Zhu. Department of Cardiothoracic Surgery, Taizhou Hospital of Zhejiang Province, Zhejiang University \\ School of Medicine, Taizhou 317000, China. Email: jianfei051@163.com; zhucc@enzemed.com. \\ Provenance and Peer Review: This article was commissioned by the Editorial Office, fournal of Thoracic Disease. The article did not undergo external \\ peer review.
}

Submitted Feb 10, 2020. Accepted for publication Feb 28, 2020.

doi: $10.21037 /$ jtd.2020.03.44

View this article at: http://dx.doi.org/10.21037/jtd.2020.03.44

\section{Introduction}

In recent years, the role of an immune checkpoint inhibitor (ICI) in tumor treatment has become increasingly prominent. Immunotherapy drugs, including PD-1, PD-L1, and CTLA-4, can prevent tumor recurrence and metastasis by blocking the cancer-derived inhibitory signal on effector T cells and removing residual cancer cells and small lesions. At present, the comprehensive treatment of lung cancer still involves surgery. Although conventional postoperative adjuvant chemotherapy or neoadjuvant chemotherapy can benefit patients and improve long-term survival rates, the patient outcome is not satisfactory (1). This has further stimulated people's research on the efficacy and safety of immunotherapy of malignant tumors.

\section{Immunotherapy in advanced non-small cell lung cancer (NSCLC)}

T-cell checkpoint inhibition has become an important treatment option for patients with NSCLC. Several studies have confirmed that ICI has better efficacy and lower toxicity in advanced NSCLC compared to chemotherapy (2-6). The KEYNOTE 024 study showed that the use of pembrolizumab in advanced NSCLC patients had significantly better progression-free survival (PFS), overall survival (OS), and objective response rate (ORR) compared to chemotherapy (2). In addition to immunotherapy alone, experts have also studied the efficacy of immunotherapy along with chemotherapy and dual immunotherapy in advanced NSCLC. The KEYNOTE 407, IMPOWER 130, and IMPOWER 150 studies showed that immunotherapy plus chemotherapy significantly improved patients' PFS and OS (3-5). The results of the CHECKMATE 227 study revealed that the PFS of immunotherapy-drug therapy was significantly longer compared to that of chemotherapy in NSCLC patients (6). The efficacy of PD-1/PD-L1 inhibitors in the adjuvant treatment of locally advanced NSCLC patients has also been verified. PACIFIC (7) phase III clinical trial confirmed that the use of durvalumab adjuvant therapy after concurrent chemoradiotherapy could significantly prolong patients' OS. Durvalumab is the only phase III immunotherapy drug recommended by the current guidelines.

\section{Neoadjuvant immunotherapy in resectable NSCLC}

The successful application of immunotherapy in patients with advanced lung cancer has inspired researchers to apply immunotherapy to patients with early lung cancer. Neoadjuvant immunotherapy has been validated in some animal experiments. A study on a mouse model of breast cancer showed that, compared with adjuvant therapy, antiPD-1 treatment before the removal of the primary tumor improved the overall survival rate of mice and produced a stronger tumor-specific CD8 $+\mathrm{T}$ cell response (8). In other malignancies, such as melanoma and glioma, studies also showed that neoadjuvant immunotherapy offered more 
significant overall survival benefits compared to adjuvant therapy $(9,10)$.

Several clinical trials of PD-1/PD-L1 inhibitors in neoadjuvant therapy have been carried out at home and abroad. These experiments have focused on the effectiveness of immunotherapy methods, such as the pathological response rate and objective response rate. However, data on safety and feasibility after surgical resection are still scarce. A clinical trial of the neoadjuvant nivolumab in patients with resectable NSCLC, published recently in the JTCVS, evaluated the efficacy of nivolumab in patients with surgically resectable NSCLC (11). A total of 22 untreated patients with stage I-IIIA NSCLC were included in this study. Two cycles of nivolumab $(3 \mathrm{mg} / \mathrm{kg})$ neoadjuvant therapy were given before surgical resection 4 and 2 weeks before the surgery, respectively. The primary study endpoints were the safety and feasibility of nivolumab neoadjuvant immunotherapy in patients with resectable early NSCLC.

Of the 22 patients included, one had a histological subtype of small cell lung cancer. Other histological subtypes included adenocarcinoma [14/21 (67\%)], squamous carcinoma [5/21 (24\%)], pleomorphic lung cancer [1/21 $(5 \%)]$, and adenosquamous carcinoma [1/21 (5\%)]. One patient had a bronchial invasion that could not be resolved. Twenty patients eventually underwent surgical resection. A total of 15 patients underwent lobectomy, two underwent pneumonectomy, one underwent lobectomy, one underwent sleeve resection, and one underwent wedge resection. Seven of the $13(54 \%)$ patients who attempted the surgery via TV-assisted thoracoscopic surgery or robotics switched to thoracotomy. However, after the analysis, the researchers found that most patients who switched to open thoracotomy experienced more severe invasion and accumulation of hilum and mediastinal lymph nodes because of inflammation caused by hilar and mediastinal lymph nodes and fibrosis, which roughly explained the objective reason for switching to thoracotomy midway through the analysis. The analysis showed that the switch might not be related to the use of neoadjuvant immune drugs. Statistical analysis of postoperative complications and recurrence rates found that 10 of the 20 patients $(50 \%)$ had complications. The most common postoperative complication was atrial arrhythmia [6/20 (30\%)]. The pathological analysis after surgical resection showed that the major pathological response (MPR: significant pathological response, which refers to the proportion of cancer cells in the resected tumors and lymph nodes below $10 \%$ ) reached $45 \%$. Eight patients
(40\%) experienced a decline in pathological stage, and two patients had complete pathological remission. This ratio was almost twice the MPR of neoadjuvant chemotherapy reported in previous studies. Eighteen months after the surgery, the patients' recurrence-free survival reached an exciting $73 \%$. Researchers also did not observe increased perioperative patient mortality and disease recurrence rates postoperatively. Although more than half of the cases of $\mathrm{TV}$-assisted thoracoscopic surgery/robot surgery turned to thoracotomy halfway through the operation during the analysis and follow-up described above, it was found that neoadjuvant therapy of nivolumab was not associated with unexpected perioperative complications or mortality. The results preliminarily demonstrated the feasibility and safety of neoadjuvant nivolumab monotherapy in resectable early NSCLC and provided a useful reference for subsequent related studies. However, this experiment had many limitations. It was only a single-arm Phase I experiment that included only 20 cases. Although the MPR rate of this experiment reached $45 \%$, it was not entirely valid. The findings need to be verified in a larger sample to determine whether it would achieve the same MPR rate. Although it was proved that immune drugs were unrelated to the high rate of thoracotomy in the experiment, we still need to explore further how to reduce the halfway thoracotomy rate and improve the safety of surgical procedures.

Several phase II trials of neoadjuvant immunotherapy have been carried out at home and abroad (Table 1). The largest of these phase II trials is the (NCT02927301) trial, which evaluated the efficacy and safety of neoadjuvant treatment of Atezolizumab in patients with IB, II, IIIA, and partially selectively resectable stage IIIB NSCLC (12). In this study, MPR was used as the primary endpoint. The analysis recruited 101 patients, 90 of whom underwent surgery. Among the 77 patients who were adequately evaluated, the pathological complete response (PCR) ratio was $5.2 \%$ (4/77), and the MPR ratio reached $19.5 \%(15 / 77)$. However, the MPR proportion in the PD-L1 negative and PD-L1 positive patients was not statistically different, and the difference in TMB in the patients with MPR and those without MPR was also non-significant. In addition to the immunotherapy model, some scholars have also explored the effects of immunotherapy plus chemotherapy for neoadjuvant therapy. For example, the NADIM trial from Spain evaluated the efficacy of neoadjuvant chemotherapy plus nivolumab immunotherapy in patients with resectable stage IIIA NSCLC (13). A total of 46 patients were included 
Table 1 Results of Phase II clinical trials of neoadjuvant immune checkpoint inhibitors for resectable small cell lung cancer mentioned in the article

\begin{tabular}{|c|c|c|c|c|c|c|c|c|c|}
\hline & Trail & Size & Phase & Treatment & $\begin{array}{l}\text { Primary } \\
\text { Endpoint }\end{array}$ & MPR (\%) & PCR (\%) & PR (\%) & $\begin{array}{c}\mathrm{AE} \\
(\text { Grade } \geq 3 \sim 4)\end{array}$ \\
\hline \multirow[t]{2}{*}{$\begin{array}{l}\text { Mono- } \\
\text { therapy }\end{array}$} & NCT02259621 & 22 & I-IIIA & Nivolumab & $\begin{array}{l}\text { Safety \& } \\
\text { feasibility }\end{array}$ & $45(9 / 20)$ & $15(3 / 20)$ & $10(2 / 21)$ & 1 \\
\hline & NCT02927301 & 101 & $\begin{array}{l}\text { IB-section; } \\
\text { resectable } \\
\text { IIIB }\end{array}$ & Atezolizumab & MPR & $19.5(15 / 77)$ & $5(4 / 77)$ & $7(6 / 90)$ & 6 \\
\hline $\begin{array}{l}\text { Combined } \\
\text { therapy }\end{array}$ & NCT02716038 & 18 & Ib-IIIa & $\begin{array}{l}\text { Atezolizumab + } \\
\text { nab-paclitaxe and } \\
\text { carboplatin }\end{array}$ & MPR & $50(7 / 14)$ & $21(3 / 14)$ & $57(8 / 14)$ & 12 \\
\hline Domestic & $\begin{array}{c}\text { ChiCTR- } \\
\text { OIC-17013726 }\end{array}$ & 40 & IB-IIIA & Sintilimab & $\mathrm{AE}$ & $40.5(15 / 37)$ & $16.2(6 / 37)$ & $20(8 / 40)$ & 4 \\
\hline
\end{tabular}

MPR, major pathological response; PCR, complete pathological response; PR, partial response; PFS, progression-free survival; AE, adverse event.

in the study. Nivolizumab + paclitaxel + carboplatin was given in three cycles before the surgery, which was performed 3 or 4 weeks after the 21 st day of the third cycle, followed by adjuvant treatment with nivolizumab for one year. The results of the study reported at the 2019 annual meeting of the American Society of Clinical Oncology showed that $85.4 \%(35 / 41)$ of patients achieved MPR, and 71.4\% (25/35) of patients achieved PCR after neoadjuvant immunotherapy plus chemotherapy. Similar trials included NCT02716038 (14), in which Atezolizumab was combined with the same carboplatin and paclitaxel, showing that the MPR rate increased to more than $50 \%$ after two cycles of neoadjuvant immunochemotherapy. In addition, the NEOSTAR trial, which was a randomized controlled study, compared the efficacy of nivolumab monotherapy with nivolumab plus Ipilimumab dual immune neoadjuvant therapy in patients with resectable NSCLC. The latest research results confirmed the 33\% MPR rate of nivolumab plus ipilimumab group, compared with $17 \%$ in the monotherapy group. A domestic PD-1 inhibitor, Sintilimab, has been proven to be effective and safe in clinical studies as neoadjuvant immunotherapy for the resection of NSCLC. Forty patients were included in the study, all of whom received two cycles (Day 1 \& Day 22) of Sintilimab before the surgery. The latest results showed that patients have an exciting MPR of $40.5 \%$. As a domestic drug in China, it has a promising future. Compared to the popular molecular targeted therapies in previous years, the results of several clinical trials on EGFR mutation inhibitor TKI have shown that after applying TKI drugs, the overall ORR rate of patients can be more than $50 \%(15,16)$. Other molecular target studies on drugs, such as EML4-ALK fusion gene inhibitors, RAF mutation inhibitors, ROS1 ectopic, BRAF mutation inhibitors, and HER-2 mutation inhibitors, have all shown significant increases in ORR, PFS, and OR. Although molecular targeted drugs have enormous benefits for patients, the increased resistance to these drugs would challenge tumor treatment. Although in recent years, a greater number of researchers have designed trials that are encouraging, they are still small phase II clinical trials, and the safety of preoperative use of checkpoint inhibitors still needs to be confirmed in more extensive phase III trials. Nevertheless, some vital phase III clinical studies have been carried out at home and abroad (Table 2). The completed and ongoing series of data show that neoadjuvant immunotherapy is a safe and effective treatment strategy for patients with early resectable lung cancer. 
Table 2 Phase III clinical studies of neoadjuvant immunotherapy

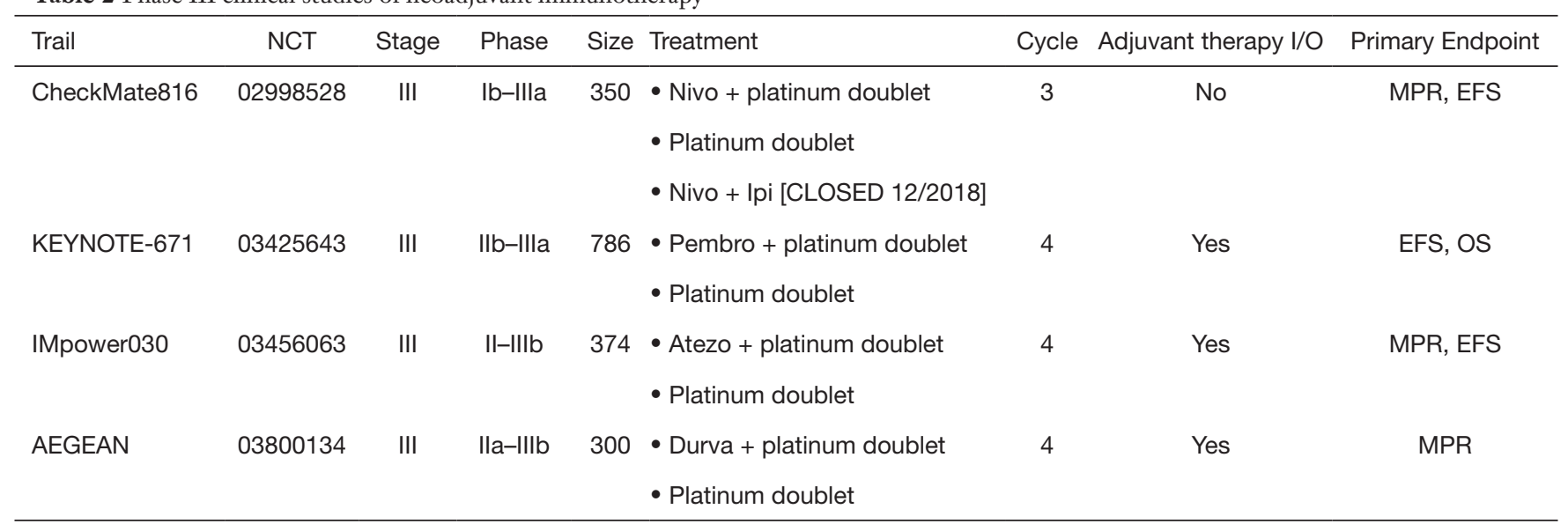

MPR, major pathological response; EFS, event-free survival; OS, overall survival.

\section{Problems with neoadjuvant immunotherapy}

Current studies have preliminarily confirmed the safety and effectiveness of neoadjuvant immunotherapy. Overall, neoadjuvant immunotherapy can induce significant pathological remissions, and it has the potential for continued anti-tumor immunity. However, many problems remain unsolved in neoadjuvant therapy with ICIs.

First, the choice of neoadjuvant immunotherapy is problematic. Choosing immune drugs is our first concern, as different ICIs contribute to a significant difference in the MPR rates. At the same time, determining the dose of the use of immune drugs, agreeing on the specific timing of using immune drugs before surgery, and deciding on whether to use immune drug adjuvant therapy after surgery still need to be verified to create the implementation guide. In the above NADIM trial, the combination of immune and chemotherapy regimens has reached a new high MPR rate for patients, which suggests that we should focus on ICIs and chemotherapy regimens. Therefore, our future research will explore immune drugs and chemotherapy drugs that can be used in combination to achieve better, pathologically significant, remission rates. Second, although ICIs have achieved excellent results in patients with different stages of NSCLC, no specific trials are currently available to prove the benefits of immunotherapy for patients in different stages of NSCLC.

Second, the preoperative evaluation of neoadjuvant immunotherapy is challenging. Whether early-stage NSCLC requires immunotherapy after surgery also needs further experimental verification. No verifiable experimental data are currently available to compare the recurrence rate and long-term survival time between surgical and nonsurgical patients after applying ICIs. At the same time, the timing of surgery after immunotherapy should still be investigated. However, from the above experiments, we know that the imaging and pathological remission rate assessments do not match after the patient is treated with immunologic drugs. Thus, the operator cannot accurately judge the patient's disease progression and may choose the incorrect scope of surgical resection, which would cause a deviation in treatment. Therefore, an accurate assessment of the degree of tumor progression before surgery is crucial for the choice of surgical method.

Third, the pharmacological effects of neoadjuvant immunotherapy are uncertain. In the above studies, some researchers have combined immunotherapy with chemotherapeutics or immunotherapy with another immunotherapy. Different treatment methods will lead to different outcomes for patients. Combination therapy can improve efficacy but may increase pharmacological toxicity and cause adverse immunotherapy outcomes. Hence, we need to find suitable markers, such as immunological markers, laboratory markers, and others, to determine the toxicity of drugs that may lead to poor prognosis and design suitable immunotherapy combined with traditional treatment.

Fourth, the unification of major pathological remission (MPR) evaluation standards is problematic. In the current studies, MPR was used as an important outcome indicator to evaluate the efficacy of immunotherapy. However, no unified standard for evaluating MPR has been established. After receiving neoadjuvant immunotherapy, the tumor, 
cells, and matrix surrounding the tumor, as well as the surrounding environment of the tumor, have all changed, having a large amount of necrotic tissue and lymphocytes. Therefore, it is necessary to develop a new standard. At present, the semi-quantitative assessment of the degree of residual cancer, that is, the ratio of the cross-sectional area of the parenchymal lesion of the surviving tumor to the cross-sectional area of the tumor bed, has been used internationally. The cross-sectional area of the tumor bed is defined as the range of tumor parenchymal cell range + necrotic cells + interstitial region. It has also been suggested that a semi-quantitative assessment of the extent of residual cancer in the lymph nodes should be conducted. In addition to the MPR standard, we should look for other alternative outcome indicators to better evaluate the critical endpoints of patients with NSCLC.

Fifth, unified indicators for the selection of beneficiaries has not been established. Although ICIs achieved exciting results in early NSCLC, the NADIM test and LCMC3 test have shown no statistical difference in the proportion of patients with MPR between PD-L1 negative and PDL1 positive patients. However, the expression level of PDL1 in the NEOSTAR test was related to imaging and pathological remission. Patients benefit more from higher PD-L1 expression. In the LCMC3 trial, there was no significant difference in TMB between patients with and without MPR. However, the results of the CheckMate 159 study showed that patients who achieved MPR had higher TMB compared to patients who did not. After the application of immunotherapy, neoantigen-specific $\mathrm{T}$ cell clones proliferated, and CD8 + T cell expression increased. This reminds us that there is no unified standard for the selection of immune drug application populations, which should be studied in subsequent experiments.

Finally, although many problems in neoadjuvant immunotherapy remain unsolved, we know from the existing research that neoadjuvant immunotherapy could attain effective pathological remission, prolong the survival time of patients, and improve the effectiveness and safety of the treatment. In future clinical trials, there are more mechanisms of neoadjuvant immunotherapy waiting for us to discover.

\section{Acknowledgments}

The authors acknowledge the MSD China Holding co., ltd. General Building Zhejiang Foreign Econnomy \& Trade Plaza, Hangzhou, China for reviewing and proofreading.

\section{Funding: None.}

\section{Footnote}

Conflicts of Interest: All authors have completed the ICMJE uniform disclosure form (available at http://dx.doi. org/10.21037/jtd.2020.03.44). The authors have no conflicts of interest to declare.

Ethical Statement: The authors are accountable for all aspects of the work in ensuring that questions related to the accuracy or integrity of any part of the work are appropriately investigated and resolved.

Open Access Statement: This is an Open Access article distributed in accordance with the Creative Commons Attribution-NonCommercial-NoDerivs 4.0 International License (CC BY-NC-ND 4.0), which permits the noncommercial replication and distribution of the article with the strict proviso that no changes or edits are made and the original work is properly cited (including links to both the formal publication through the relevant DOI and the license). See: https://creativecommons.org/licenses/by-nc-nd/4.0/.

\section{References}

1. Nagasaka M, Gadgeel SM. Role of chemotherapy and targeted therapy in early-stage non-small cell lung cancer. Expert Rev Anticancer Ther 2018;18:63-70.

2. Reck M, Rodríguez-Abreu D, Robinson AG, et al. Pembrolizumab versus Chemotherapy for PD-L1Positive Non-Small-Cell Lung Cancer. N Engl J Med 2016;375:1823-33.

3. Paz-Ares L, Luft A, Vicente D, et al. Pembrolizumab plus Chemotherapy for Squamous Non-Small-Cell Lung Cancer. N Engl J Med 2018;379:2040-51.

4. Cappuzzo F, McCleod M, Hussein M, et al. IMpower130: Progression-free survival (PFS) and safety analysis from a randomised phase III study of carboplatin + nab-paclitaxel $(\mathrm{CnP})$ with or without atezolizumab (atezo) as first-line (1L) therapy in advanced non-squamous NSCLC. Ann Oncol 2018;29 Suppl 8:viii742-3.

5. Socinski MA, Jotte RM, Cappuzzo F, et al. Atezolizumab for First-Line Treatment of Metastatic Nonsquamous NSCLC. N Engl J Med 2018;378:2288-301.

6. Hellmann MD, Ciuleanu TE, Pluzanski A, et al. Nivolumab plus Ipilimumab in Lung Cancer with a High Tumor Mutational Burden. N Engl J Med 
2018;378:2093-104.

7. Antonia SJ, Villegas A, Daniel D, et al. Durvalumab after chemoradiotherapy in stage III non-small-cell lung cancer. N Engl J Med 2017;377:1919-29.

8. Liu J, Blake SJ, Yong MC, et al. Improved efficacy of neoadjuvant compared to adjuvant immunotherapy to eradicate metastatic disease. Cancer Discov 2016;6:1382-99.

9. Blank CU, Rozeman EA, Fanchi LF, et al. Neoadjuvant versus adjuvant ipilimumab plus nivolumab in macroscopic stage III melanoma. Nat Med 2018;24:1655-61.

10. Cloughesy TF, Mochizuki AY, Orpilla JR, et al. Neoadjuvant anti-PD-1 immunotherapy promotes a survival benefit with intratumoral and systemic immune responses in recurrent glioblastoma. Nat Med 2019;25:477-86.

11. Bott MJ, Yang SC, Park BJ, et al. Initial results of pulmonary resection after neoadjuvant nivolumab in patients with resectable non-small cell lung cancer. J

Cite this article as: Ren S, Wang C, Shen J, Zhu C. Neoadjuvant immunotherapy with resectable non-small cell lung cancer: recent advances and future challenges. J Thorac Dis 2020;12(4):1615-1620. doi: 10.21037/jtd.2020.03.44
Thorac Cardiovasc Surg 2019;158:269-76.

12. Kwiatkowski DJ, Rusch VW, Chaft JE, et al. Neoadjuvant atezolizumab in resectable non-small cell lung cancer (NSCLC): interim analysis and biomarker data from a multicenter study (LCMC3). J Clin Oncol 2019;37:8503.

13. Provencio-Pulla M, Nadal-Alforja E, Cobo M, et al. Neoadjuvant chemo/immunotherapy for the treatment of stages IIIA resectable non-small cell lung cancer (NSCLC): A phase II multicenter exploratory study-NADIM studySLCG. J Clin Oncol 2018;36:8521.

14. Shu CA, Grigg C, Chiuzan C, et al. Neoadjuvant atezolizumab+ chemotherapy in resectable non-small cell lung cancer (NSCLC). J Clin Oncol 2018;36:8532.

15. JännePA, Yang JC, Kim DW, et al. AZD9291 in EGFR inhibitor-resistant non-small-cell lung cancer. N Engl J Med 2015;372:1689-99.

16. Sequist LV, Soria JC, Goldman JW, et al. Rociletinib in EGFR-mutated non-small-cell lung cancer. N Engl J Med 2015;372:1700-9. 